\title{
Chemistry and line emission from evolving Herbig Ae disks
}

\author{
B. Jonkheid ${ }^{1}$, C. P. Dullemond ${ }^{2}$, M. R. Hogerheijde ${ }^{1}$, and E. F. van Dishoeck ${ }^{1}$ \\ ${ }^{1}$ Sterrewacht Leiden, Leiden University, PO Box 9513, 2300 RA Leiden, The Netherlands \\ e-mail: jonkheid@strw. leidenuniv.nl \\ 2 Max Planck Institut für Astronomie, Königstuhl 17, 69117 Heidelberg, Germany
}

Received 23 May 2006 / Accepted 28 October 2006

\begin{abstract}
Aims. To calculate chemistry and gas temperature of evolving protoplanetary disks with decreasing mass or dust settling, and to explore the sensitivity of gas-phase tracers.

Methods. The density and dust temperature profiles for a range of models of flaring and self-shadowed disks around a typical Herbig Ae star are used together with 2-dimensional ultraviolet (UV) radiative transfer to calculate the chemistry and gas temperature. In each model the line profiles and intensities for the fine structure lines of [O I],$[\mathrm{C} \mathrm{II}]$ and $[\mathrm{CI}$ ] and the pure rotational lines of $\mathrm{CO}, \mathrm{CN}$, $\mathrm{HCN}$ and $\mathrm{HCO}^{+}$are determined.

Results. The chemistry shows a strong correlation with disk mass. Molecules that are easily dissociated, like HCN, require high densities and large extinctions before they can become abundant. The products of photodissociation, like $\mathrm{CN}$ and $\mathrm{C}_{2} \mathrm{H}$, become abundant in models with lower masses. Dust settling mainly affects the gas temperature, and thus high temperature tracers like the $\mathrm{O}$ and $\mathrm{C}^{+}$fine structure lines. The carbon chemistry is found to be very sensitive to the adopted PAH abundance. The line ratios $\mathrm{CO} /{ }^{13} \mathrm{CO}, \mathrm{CO} / \mathrm{HCO}^{+}$and [O I] $63 \mu \mathrm{m} / 146 \mu \mathrm{m}$ can be used to distinguish between disks where dust growth and settling takes place, and disks that undergo overall mass loss.
\end{abstract}

Key words. astrochemistry - stars: circumstellar matter - stars: planetary systems: protoplanetary disks

\section{Introduction}

Circumstellar disks are a natural by-product of the formation of stars of low to intermediate masses, the former objects being identified as T-Tauri stars while the latter are Herbig Ae/Be stars. From models of the spectral energy distributions (SEDs) at infrared and millimeter wavelengths, which track the dust emission from these disks, it has been concluded that some disks have a flaring geometry, allowing them to intercept a significant portion of the stellar radiation and re-emitting it at longer wavelengths (e.g. Kenyon \& Hartmann 1987; Chiang \& Goldreich 1997). In a recent study, Dullemond \& Dominik (2004) suggested that the difference between flaring ("group I") and flat ("group II") disks can be explained by the presence of a puffedup inner rim that casts a shadow over the disk. Depending on whether there is enough material in the outer disk to rise above the shadow either an illuminated flared disk or a self-shadowed flat disk results. One interpretation of the occurrence of group I and group II sources is that flared disks evolve into flat disks when the dust grows and settles to the midplane, or when the disk's mass decreases due to dissipation and evaporation.

While SEDs are useful to examine the properties of the dust content of disks, the physical and chemical properties of the gas are studied through line emission from both fine-structure transitions in atoms and rotational and vibrational transitions in molecules. The pure rotational lines and ro-vibrational lines of CO (e.g. Koerner \& Sargent 1995; Dutrey et al. 1997; Thi et al. 2001; Brittain et al. 2003; Dent et al. 2005), and $\mathrm{H}_{2}$ (e.g. Bary et al. 2003) have been observed in disks, as well as the rotational lines of several other molecules like $\mathrm{CN}, \mathrm{HCN}, \mathrm{HCO}^{+}$and $\mathrm{C}_{2} \mathrm{H}$ (e.g. Dutrey et al. 1997; Thi et al. 2004). To obtain information on the temperature, chemical composition and total mass of the gas from the observed emission lines, the chemistry of the disk has to be modelled. In recent years calculations of both the chemistry (e.g. Aikawa et al. 1997; van Zadelhoff et al. 2003) and the gas temperature (e.g. Jonkheid et al. 2004; Kamp \& Dullemond 2004) have been performed using a density structure based on previous models of the disk structure (e.g. D'Alessio et al. 1998; Dullemond et al. 2002). In these models, the density structure is calculated assuming vertical hydrostatic equilibrium, and the dust temperature is obtained by solving the radiative transfer of stellar light. In most cases the gas temperature is assumed to be equal to the dust temperature, but some models calculate the gas temperature and density structure in equilibrium with each other (e.g. Gorti \& Hollenbach 2004; Nomura \& Millar 2005). The emission lines are modelled from the results of the chemistry and temperature calculations (e.g. Kamp et al. 2003; Jonkheid et al. 2004; Gorti \& Hollenbach 2004). Effects of grain growth and dust settling on the chemistry have also been considered (e.g. Kamp et al. 2003; Jonkheid et al. 2004, 2006; Aikawa \& Nomura 2006).

In the current paper calculations of the chemistry and gas temperature are presented based on the disk structure models of group I and group II sources by Dullemond \& Dominik (2004) to investigate how the chemistry and line emisison change in a series of evolving disks. Special attention was given to identify possible tracers of the disk geometry that can supplement and confirm the information gained from SEDs. This work complements the calculations for optically thin disks by Kamp et al. (2006). 
Table 1. Model parameters used in the calculations. Note that the dust masses given here include only small (interstellar sized) grains.

\begin{tabular}{lll}
\hline \hline Model & $M_{\text {gas }}\left(M_{\odot}\right)$ & $M_{\text {dust }}\left(M_{\odot}\right)$ \\
\hline B1 & $10^{-1}$ & $10^{-3}$ \\
B2 & $10^{-2}$ & $10^{-4}$ \\
B3 & $10^{-3}$ & $10^{-5}$ \\
B4 & $10^{-4}$ & $10^{-6}$ \\
\hline BL1 & $10^{-1}$ & $10^{-3}$ \\
BL2 & $10^{-1}$ & $10^{-4}$ \\
BL3 & $10^{-1}$ & $10^{-5}$ \\
BL4 & $10^{-1}$ & $10^{-6}$ \\
\hline
\end{tabular}

\section{Model}

The chemistry is calculated for a range of disk structure models by Dullemond \& Dominik (2004) in which the disk mass and dust/gas ratio are varied systematically (see Table 1 and Figs. 1 and 2). In that paper the vertical structure of the disk is computed in hydrostatic equilibrium assuming several radial distributions and total disk masses. In all models the gas temperature was taken equal to the dust temperature. Under consideration here are models B1-B4 and BL1-BL4. These models have a surface density distribution that falls off as $\Sigma \propto R^{-1.5}$ for $R<200 \mathrm{AU}$; beyond $200 \mathrm{AU}$ the surface density drops off as $\Sigma \propto R^{-12}$ to simulate an effective outer edge of $200 \mathrm{AU}$ while keeping a continuous mass distribution. In the B series of models the total disk mass is varied from $10^{-1} M_{\odot}$ in B1 to $10^{-4} M_{\odot}$ in B4, while keeping a constant dust/gas mass ratio of 0.01 . In the BL series the gas mass stays constant at $10^{-1} M_{\odot}$ and the dust/gas ratio is varied from 0.01 in BL1 to $10^{-5}$ in BL4. Dullemond \& Dominik (2004) kept the overall dust/gas ratio in the BL series constant at 0.01 by removing the interstellar-sized grains $(\sim 0.1 \mu \mathrm{m})$ from the disk and replacing them with $\mathrm{cm}$-sized grains in the midplane, thus simulating the effects of dust settling and growth, but these large grains are excluded from the chemical calculations presented here because of their very limited influence (e.g. Kamp \& van Zadelhoff 2001; Jonkheid et al. 2006). It should be noted that models B1 and BL1 are identical. The abundance of polycyclic aromatic hydrocarbons (PAHs) is assumed to follow the dust/gas ratio (i.e. the PAH abundance drops off with a factor 10 in each step of the BL series), consistent with the findings of Acke \& van den Ancker (2004) and Habart et al. (2004) that PAH emission is weaker in group II sources than in group I sources. The abundances of the elements used in the calculations, given in Table 2, are representative for disks where $\mathrm{N}$ and $\mathrm{O}$ are mostly depleted in some form (e.g. $\mathrm{H}_{2} \mathrm{O}$ ice) onto dust rains.

The densities and dust temperatures are taken directly from the Dullemond \& Dominik (2004) models. Only regions with densities $n_{\mathrm{H}}>10^{3} \mathrm{~cm}^{-3}$ were taken into account, regions with lower densities do not contribute enough column density to be taken into consideration. Note that the dust temperature does not fall below $30 \mathrm{~K}$ anywhere in the disk, so the freeze-out of CO can be disregarded. The maximum outer radius of the computational grid is taken to be $500 \mathrm{AU}$.

In the B series (Fig. 1) the disk is initially very large and flared. As the mass decreases in this series, the disk becomes smaller in both the radial and the vertical direction. Although the disk is still rather thick in models B3 and B4, the $\tau=1$ surface for stellar radiation shows that there is little flaring: the disk does not intercept much radiation at large radii. These models are a transition between group I sources (of which B1 and B2 are examples) and group II sources (B5 and B6), which have too low gas masses to be considered in this paper. The structure of the dust temperature and the radiation field show that the inner part of the disk is strongly self-shadowed. As the disk mass decreases, this effect becomes less strong and the low dust temperatures and UV intensities are confined to a narrow region near the midplane.

The BL series is very similar to the B series regarding the temperature structure and UV intensities. While one would expect more flaring in models BL3 and BL4 (since the gas distribution is higher in the outer disk), the $\tau=1$ surface again shows that these disks have a largely flat geometry. As in the B series, these models form a transition between group I (BL1 and BL2) and group II (BL5 and BL6) sources.

The transfer of UV radiation is performed with the 2-D Monte Carlo code by van Zadelhoff et al. (2003) to account for the effects of forward scattering and to include the interstellar radiation field. The self-shielding of $\mathrm{H}_{2}$ and $\mathrm{CO}$ are included by assuming constant abundances for these species of 0.5 and $10^{-5}$, respectively; the results do not depend strongly on the values used. The shielding factors are calculated with the mean column density computed by the Monte Carlo code (for more details, see van Zadelhoff et al. 2003). The results of the UV transfer are used to calculate the photorates directly from the cross-sections and local radiation field (following van Dishoeck 1988) and photoelectric heating rates on classical grains and PAHs (Tielens \& Hollenbach 1985; Bakes \& Tielens 1994, respectively).

For the stellar radiation field a NEXTGEN spectrum was adopted with $T_{\text {eff }}=10000 \mathrm{~K}, \log g=4$ (Hauschildt et al. 1999, see Fig. 3) and a stellar radius of $2 R_{\odot}$. The interstellar radiation field was taken from Draine (1978), with the extension for $\lambda>2000 \AA$ by van Dishoeck \& Black (1982). Since the formulation of the photoelectric heating rates does not take the detailed spectrum of the local radiation field into account, the correction factor by Spaans et al. (1994) (Eq. (13) in that paper) was used to account for the $10000 \mathrm{~K}$ stellar spectrum. The value $I_{\mathrm{UV}}$ characterizing the strength of the UV field was obtained by integrating the radiation field between 912 and $2050 \AA$ and dividing this by the same integral for the Draine (1978) field.

With the photorates and photoelectric heating rates known, the chemistry and gas temperature are calculated as outlined in Jonkheid et al. (2004): the chemical network of Jansen et al. (1995) is used to calculate the gas-phase chemistry in steady state, and the thermal balance is solved using an escape probability formalism assuming cooling radiation escapes from the disk in the vertical direction (i.e., column densities used in the escape probability are calculated vertically). The heating processes included in the calculations are: photoelectric effect on both classical grains and PAHs, cosmic ray ionization of $\mathrm{H}$ and $\mathrm{H}_{2}, \mathrm{H}_{2}$ formation, $\mathrm{H}_{2}$ photodissociation, collisional de-excitation of the excited vibrational levels of $\mathrm{H}_{2}$ and the fine structure levels of $\mathrm{O}$, and $\mathrm{C}$ photoionization. The gas is cooled by line emission from the fine structure lines of $\mathrm{C}^{+}, \mathrm{C}$ and $\mathrm{O}$, the rotational lines of $\mathrm{CO}$, and thermal coupling with dust grains. A more thorough description of these processes can be found in Jonkheid et al. (2004).

The line profiles are calculated from the chemistry and gas temperature using the 2-D cylindrical symmetric accelerated Monte Carlo code RATRAN by Hogerheijde \& van der Tak (2000). To speed up the calculations only the material outside $R=10$ AU is considered, since the inner part of the disk contributes little to the overall emission of the atomic and molecular lines under consideration in a typical submm observing beam $\left(\sim 15^{\prime \prime}\right)$. The number of grid cells varies between 900 and 1500 , depending on the model. 

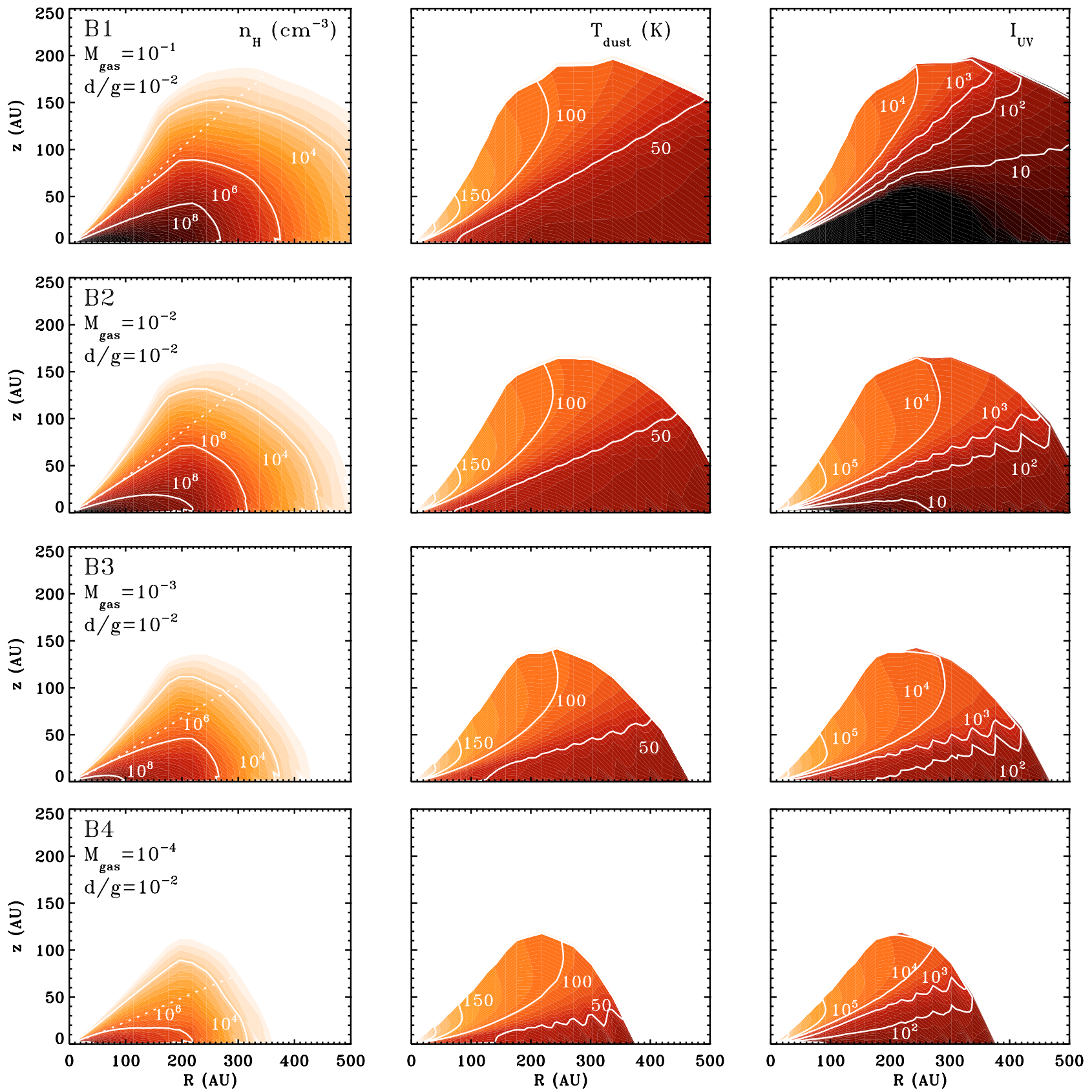

Fig. 1. Input densities (left column) dust temperatures (middle column) and integrated strength of the UV field (right column) for models B1-B4 of Dullemond \& Dominik (2004). The gas mass (in $M_{\odot}$ ) and dust/gas ratio are indicated in the for each row. The UV field is given in terms of $I_{\mathrm{UV}}$ times the strength of the interstellar radiation field by Draine (1978) integrated between 912 and $2050 \AA$. The dotted lines in the density plots give the boundary where the visual extinction towards the central star is 1 mag.

\section{Results and discussion}

\subsection{Gas temperature}

The calculated gas temperatures are illustrated in Figs. 4 and 6 for models B1-B4, and in Figs. 5 and 7 for models BL1-BL4. In the B series, the surface of the disk is very hot (several thousand $\mathrm{K}$ ) due to the photoelectric heating, which is very efficient compared to the cooling by $[\mathrm{OI}]$ and $\mathrm{Ly} \alpha$. The temperatures found here are consistent with PDR models with $n_{\mathrm{H}}=10^{3} \mathrm{~cm}^{-3}$ and $I_{\mathrm{UV}}=10^{4}-10^{5}$. The higher densities in the optically thin part of the disk below the immediate surface increase the efficiency of $[\mathrm{O} \mathrm{I}]$ cooling, resulting in lower temperatures (several hundred
$\mathrm{K})$. Deeper into the disk the densities are so high $\left(10^{7}-10^{8} \mathrm{~cm}^{-3}\right)$ that gas-dust coupling dominates the thermal balance and the gas temperature is equal to the dust temperature. In the outer parts of the disk $(R>200 \mathrm{AU})$ the densities are not high enough for gas-dust coupling to dominate due to the rapid decline of the total column density. In those regions, the gas temperature drops below the dust temperature due to efficient $\mathrm{CO}$ cooling.

It should be noted that the gas temperatures found here are higher than those reported in Jonkheid et al. (2004) for T-Tauri disks. This is because the radiation fields used in the current paper are almost two orders of magnitude larger (see Fig. 1): at a radius of $100 \mathrm{AU}$ the current models have $I_{\mathrm{UV}}=5-6 \times 10^{4}$ at the disk's surface, in contrast with a value of $5-6 \times 10^{2}$ in 

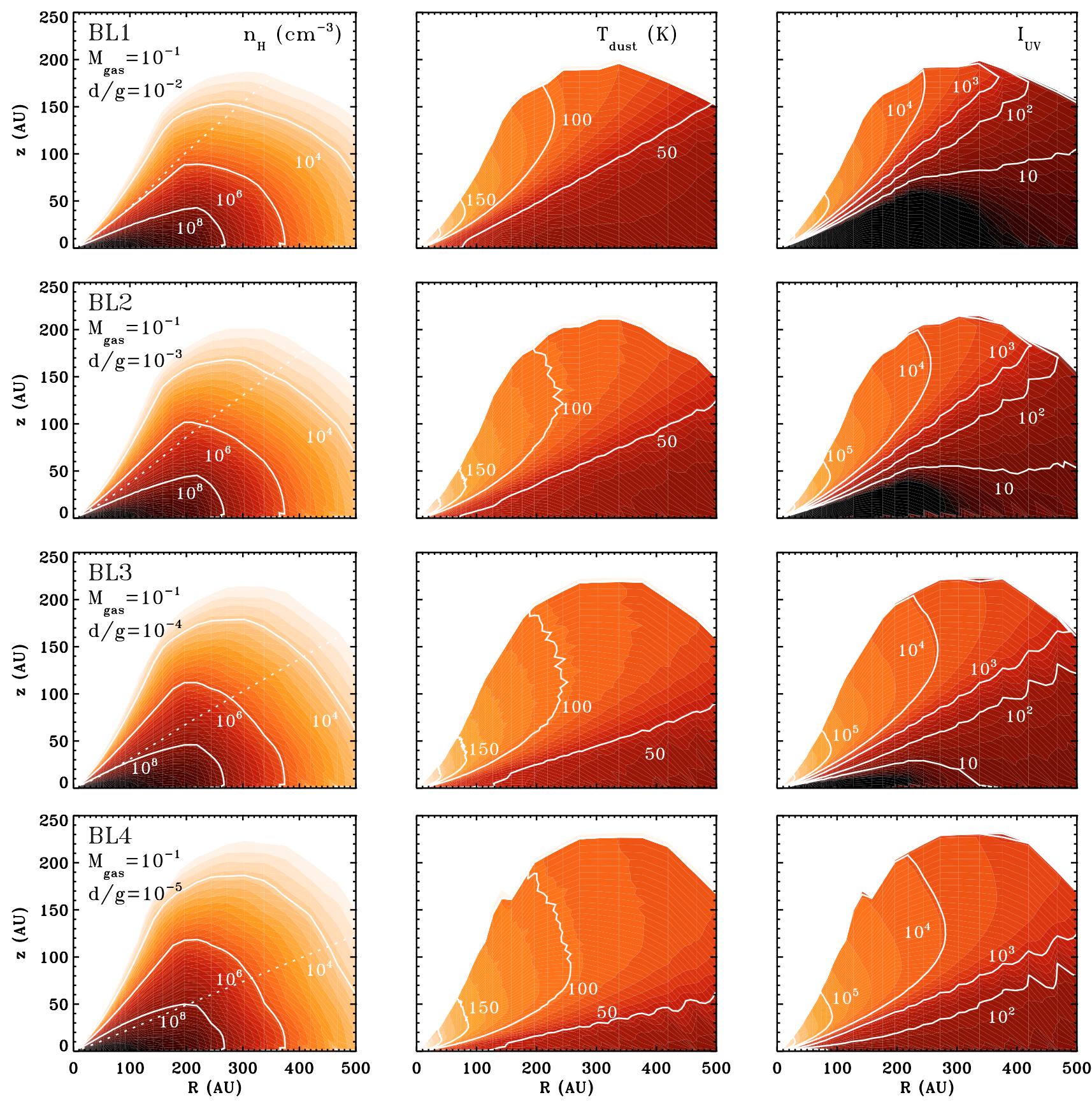

Fig. 2. As Fig. 1, but for models BL1-BL4.

Jonkheid et al. (2004). This is due to the more luminous central star used by Dullemond \& Dominik (2004) and this work.

In the BL series the importance of photoelectric heating decreases with each step because both small dust grains and PAHs disappear from the disk. This means that the total heating rate is less than in the B series, and consequently the temperatures at the disk's surface are lower (20-200 K going from BL4 to BL2). In models BL3 and BL4 heating by $\mathrm{C}$ ionization becomes important, and this heating rate has a similar magnitude as the photoelectric processes. At large depths, gas/dust coupling dominates the thermal balance, even with the low dust/gas ratio in these models. Since the gas temperature is lower than the dust temperature in much of the disk, this means that the temperature increases toward the midplane.

\subsection{Chemistry}

The vertical distribution of chemical species resembles the structure commonly found in PDRs: a thin layer of mostly atomic hydrogen at the surface, while slightly deeper in the disk $\mathrm{H}_{2}$ becomes self-shielding and thus hydrogen becomes mainly molecular. At the surface carbon is mostly in the form of $\mathrm{C}^{+}$and converts to $\mathrm{C}$ and $\mathrm{CO}$ deeper in the disk. At very high optical depths atomic oxygen is locked into $\mathrm{O}_{2}$.

There are several deviations from the classical PDR structure. In the B series there is a layer of relatively high $\mathrm{CO}$ abundance in the optically thin part of the disk (at $z \gtrsim 50 \mathrm{AU}$ ), where $\mathrm{CO}$ is not self-shielding. This is caused by the high temperatures in this layer, which help formation of $\mathrm{CO}$ via the reaction $\mathrm{C}^{+}+\mathrm{H}_{2} \rightarrow \mathrm{CH}^{+}+\mathrm{H}$, which has an enery barrier of $4600 \mathrm{~K}$. 
Table 2. Adopted gas-phase elemental abundances with respect to total hydrogen.

\begin{tabular}{ll}
\hline \hline Element & abundance \\
\hline $\mathrm{C}$ & $1.2 \times 10^{-4}$ \\
$\mathrm{O}$ & $1.6 \times 10^{-4}$ \\
$\mathrm{~N}$ & $2.0 \times 10^{-5}$ \\
$\mathrm{Mg}$ & $4.2 \times 10^{-6}$ \\
$\mathrm{~S}$ & $1.9 \times 10^{-6}$ \\
$\mathrm{Si}$ & $8.0 \times 10^{-7}$ \\
$\mathrm{Fe}$ & $4.3 \times 10^{-6}$ \\
$\mathrm{PAH}$ & $1 \times 10^{-10}-1 \times 10^{-7 a}$ \\
\hline
\end{tabular}

${ }^{a}$ In the B series of models, the PAH abundance is kept constant at $10^{-7}$. In the BL series, the PAH abundance is lowered together with the dust content of the disk, going from $10^{-7}$ in BL1 to $10^{-10}$ in BL4.

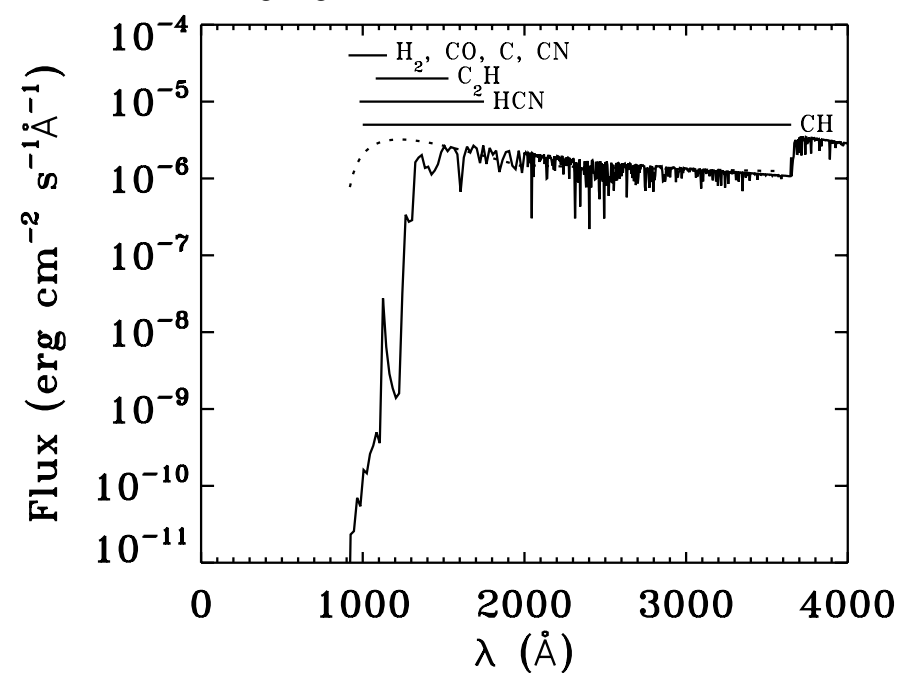

Fig. 3. The radiation fields used in the model. The solid line gives the stellar radiation field, scaled to the interstellar UV field by Draine (1978) (dotted line). The horizontal lines indicate the wavelength ranges where several important molecules are dissociated.

$\mathrm{CH}^{+}$in turn reacts with $\mathrm{O}$ to form $\mathrm{CO}^{+}$, which leads to $\mathrm{HCO}^{+}$ and $\mathrm{CO}$. Although $\mathrm{CO}$ is completely self-shielded in the midplane of most of the models (an exception being the outer regions in model $\mathrm{B} 4$, where the $\mathrm{CO}$ photodissociation by the interstellar UV field is unshielded), the abundance of $\mathrm{C}$ is still relatively high there. This is caused by the shape of the stellar radiation field (see Fig. 3): there are still many continuum photons with $\lambda>1200 \AA$ which can dissociate precursor molecules like $\mathrm{CH}$, which is an important pathway of $\mathrm{CO}$ formation. In the later steps of the BL series $\mathrm{C}$ is the dominant form of carbon in the midplane instead of CO. This is partly due to the photodissociation of precursor molecules, and partly because the formation of $\mathrm{CO}$ is further suppressed due to lack of PAHs in these models. The reaction $\mathrm{PAH}: \mathrm{H}+\mathrm{C}^{+} \rightarrow \mathrm{PAH}+\mathrm{CH}^{+}$(where $\mathrm{PAH}: \mathrm{H}$ is a $\mathrm{PAH}$ with an extra $\mathrm{H}$ atom) is another important step in the reaction pathway leading to $\mathrm{CO}$ (for rate coefficient, see Canosa et al. 1995). In the later steps of the BL series the PAH abundance is very low, and so this pathway is closed.

The global distributions of $\mathrm{CO}$ and $\mathrm{HCN}$ are illustrated in Fig. 4 for models B1-B4, and in Fig. 5 for models BL1-BL4. The abundances of $\mathrm{CO}$ and $\mathrm{HCN}$ decrease significantly going from model B1 to B4, while this effect is less pronounced going from BL1 to BL4. Although the UV continuum flux is comparable in both series, the total gas mass stays at $0.1 M_{\odot}$ in the $\mathrm{BL}$ series while it decreases with factors of 10 in the B series.
The self-shielding of $\mathrm{CO}$ will therefore be much stronger in the $\mathrm{BL}$ series, resulting in higher $\mathrm{CO}$ abundances. $\mathrm{HCN}$ is rapidly dissociated in the optically thin regions of both series, but its formation rate correlates with $\mathrm{CO}$ abundance. Therefore, a rapid decrease in $\mathrm{HCN}$ abundance can be seen in the $\mathrm{B}$ series, where there is not enough $\mathrm{CO}$ to counter the rapid photodissociation. In the BL series, however, the high $\mathrm{CO}$ abundances increase the production rate of $\mathrm{HCN}$, keeping it at a higher abundance than in the B series.

A more detailed view of the chemistry is given in Figs. 6 and 7, which show a vertical cut at 200 AU. In the B series the high $\mathrm{CO}$ abundances at the surface result in high abundances of $\mathrm{HCO}^{+}, \mathrm{C}_{2} \mathrm{H}, \mathrm{CN}$ and $\mathrm{HCN}$. The abundance of $\mathrm{C}_{2} \mathrm{H}$ follows the same trends as $\mathrm{CN}$, which is in agreement with the results by van Zadelhoff et al. (2003) for T-Tauri stars. Note that in contrast with van Zadelhoff et al. (2003) (who found similar abundances for these molecules) the abundance of $\mathrm{C}_{2} \mathrm{H}$ found here is one to two orders of magnitude less than $\mathrm{CN}$. This is due to the difference in stellar spectrum used in the current paper, which has a jump in intensity at wavelengths longer than $1200 \AA$. Since $\mathrm{C}_{2} \mathrm{H}$ can be dissociated by photons with $\lambda>1200 \AA$ and $\mathrm{CN}$ is dissociated only at $\lambda<1150 \AA$, the photodissociation rate of $\mathrm{C}_{2} \mathrm{H}$ is much higher, and thus this molecule has a lower abundance.

The same effect is visible in the abundance of $\mathrm{HCN}$ compared to $\mathrm{CN}$ : $\mathrm{HCN}$ is easily dissociated by photons with $\lambda>$ $1200 \AA$, giving it a lower abundance than $\mathrm{CN}$ in most of the disk. Only in the heavily shadowed regions near the midplane is $\mathrm{HCN}$ more abundant than $\mathrm{CN}$. In the $\mathrm{BL}$ series the densities near the midplane are high enough to increase the HCN formation rate, and so the $\mathrm{HCN}$ abundance is higher than $\mathrm{CN}$ even in the optically thin models BL3 and BL4.

The abundances of $\mathrm{HCO}^{+}$in the deeper layers of the disk are lower by several orders of magnitude than those found by van Zadelhoff et al. (2003). A possible explanation for this may be that charge exchange with metals plays a more important role in the current model, since the densities are a factor $\sim 100$ higher. Also, as for $\mathrm{CO}$, the rapid photodissociation of hydrocarbon precursors plays a role.

In summary, the B series shows a clear trend in molecular abundances: going from model B1 to B4 molecules are more easily dissociated, and the peaks in their vertical distributions occur closer to the midplane. In the BL series the trend is less clear. Going from model BL1 to BL2, the drop in gas temperature greatly affects the carbon chemistry. The differences between models BL2 to BL3 are limited, and models BL3 and BL4 are nearly identical. Again there is a shift toward the midplane in the peak of the vertical abundance distribution, in agreement with earlier findings of Jonkheid et al. (2004) and Aikawa \& Nomura (2006), but this shift is small compared to the B series.

\subsubsection{Influence of PAHs}

It is assumed that PAHs are present at interstellar abundances $\left(n_{\mathrm{PAH}} / n_{\mathrm{H}}=10^{-7}\right)$ for a dust/gas ratio of $10^{-2}$, and that these abundances scale with the dust/gas ratio. This assumption is consistent with the findings of Acke \& van den Ancker (2004) and Habart et al. (2004), although models by Geers et al. (in press) suggest an abundance that is lower by as much as an order of magnitude. The influence of PAHs on the chemistry can best be seen in the BL series (Fig. 7), where the PAH abundance is decreased by a factor 10 at each step. PAHs affect the chemistry mainly through charge exchange reactions (of the form $\mathrm{PAH}+\mathrm{X}^{+} \rightarrow \mathrm{PAH}^{+}+\mathrm{X}$ ) and hydrogenation reactions (of 

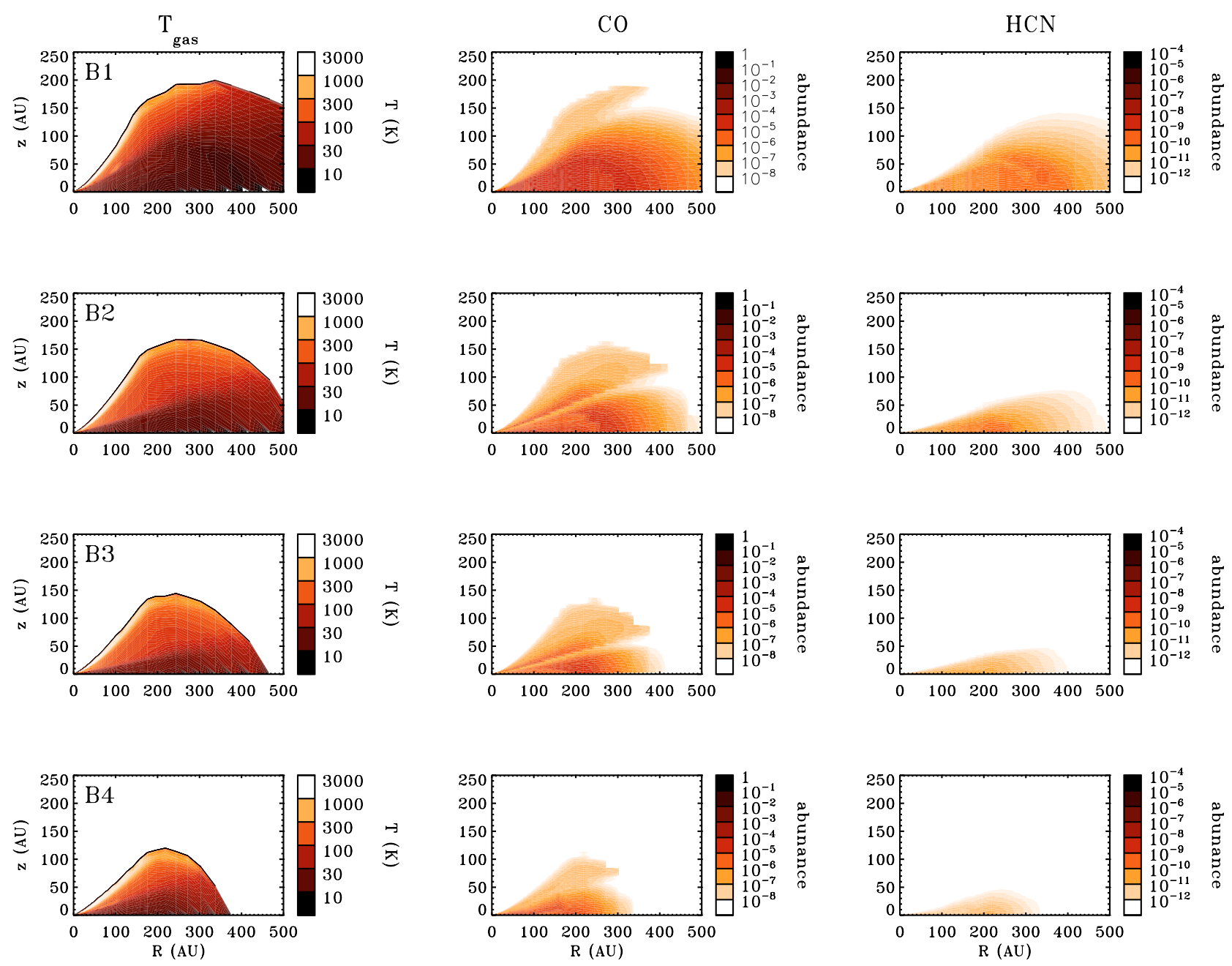

Fig. 4. Gas temperatures (left column), $\mathrm{CO}$ abundances (middle column) and $\mathrm{HCN}$ abundances (right column) for models B1-B4.

the form $\mathrm{PAH}: \mathrm{H}+\mathrm{X} \rightarrow \mathrm{PAH}+\mathrm{XH})$. Especially the latter reactions are important in the formation of molecules, as can be seen in the distribution of $\mathrm{CO}$ in the BL model series (seen in Figs. 5 and 7), where the PAH abundance is decreased in each step. As a result, the reaction $\mathrm{C}^{+}+\mathrm{PAH}: \mathrm{H} \rightarrow \mathrm{CH}^{+}+\mathrm{PAH}$ becomes less efficient; since $\mathrm{CH}^{+}$is a chemical precursor of $\mathrm{CO}$ (via $\mathrm{CO}^{+}$and $\mathrm{HCO}^{+}$), the overall $\mathrm{CO}$ abundance decreases together with the PAH abundance.

\subsubsection{Carbon chemistry}

In some of the models, particularly BL3 and BL4, there is a broad layer near the disk midplane where atomic carbon is the main carbon-bearing species. This somewhat unconventional result is caused by shape of the stellar radiation field, shown in Fig. 3: the stellar radiation has few photons in the 912-1200 $\AA$ range, most of the flux is at longer wavelengths. This means that the photoionization rate of $\mathrm{C}$ and the photodissociation rate of CO are small, since these processes occur in the 912-1100 range. The photodissociation rate of $\mathrm{CH}$ is relatively high, however, since this molecule is dissociated at longer wavelengths than $\mathrm{CO}$. Since $\mathrm{CH}$ is an important chemical precursor to $\mathrm{CO}$, the rapid dissociation of $\mathrm{CH}$ means that the formation rate of $\mathrm{CO}$ is very low, resulting in a low $\mathrm{CO}$ abundance. Since atomic carbon is released by the photodissociation of most carbon-bearing molecules, and the ionization rate of atomic carbon is very low, carbon is mostly neutral and atomic.

\subsection{Column densities}

The vertical column densities of $\mathrm{CO}, \mathrm{CN}, \mathrm{HCN}, \mathrm{C}_{2} \mathrm{H}$ and $\mathrm{HCO}^{+}$ are shown in Fig. 8. It can be seen that the greatest variations occur in the B series for $\mathrm{CO}$ and $\mathrm{HCN}$. The $\mathrm{CO}$ abundance is sensitive to the gas mass due to self-shielding. Similarly, HCN occurs only in regions that are dense and have sufficient continuum optical depth toward the central star. Both of these conditions are strongly dependent on the disk mass. The column densities of $\mathrm{CN}$ and $\mathrm{C}_{2} \mathrm{H}$ in the $\mathrm{B}$ series show a peak which starts at relatively large radii and shifts inward going from B1 to B4. These molecules require a moderate optical depth toward the central star: large enough so they and their precursors are not dissociated, but small enough that they can still be formed by photodissociation of less stable molecules $\left(\mathrm{HCN}\right.$ for $\mathrm{CN} ; \mathrm{C}_{2} \mathrm{H}_{2}$ and $\mathrm{C}_{3} \mathrm{H}$ for $\mathrm{C}_{2} \mathrm{H}$ ). The $\mathrm{HCO}^{+}$peak also shifts inward due to the lower abundance of $\mathrm{CO}$ in the outer regions of the disk.

In the BL series, the $\mathrm{CO}$ column densities show a clear distinction between on the one side models BL1 and BL2, and on the other side BL3 and BL4. The reason for this can be seen in Fig. 7: in models BL1 and BL2, CO is the main form of carbon in the dense regions near the midplane. In BL3 and BL4, $\mathrm{C}$ becomes the dominant form of carbon due to the low PAH 
$\mathrm{T}_{\text {gas }}$
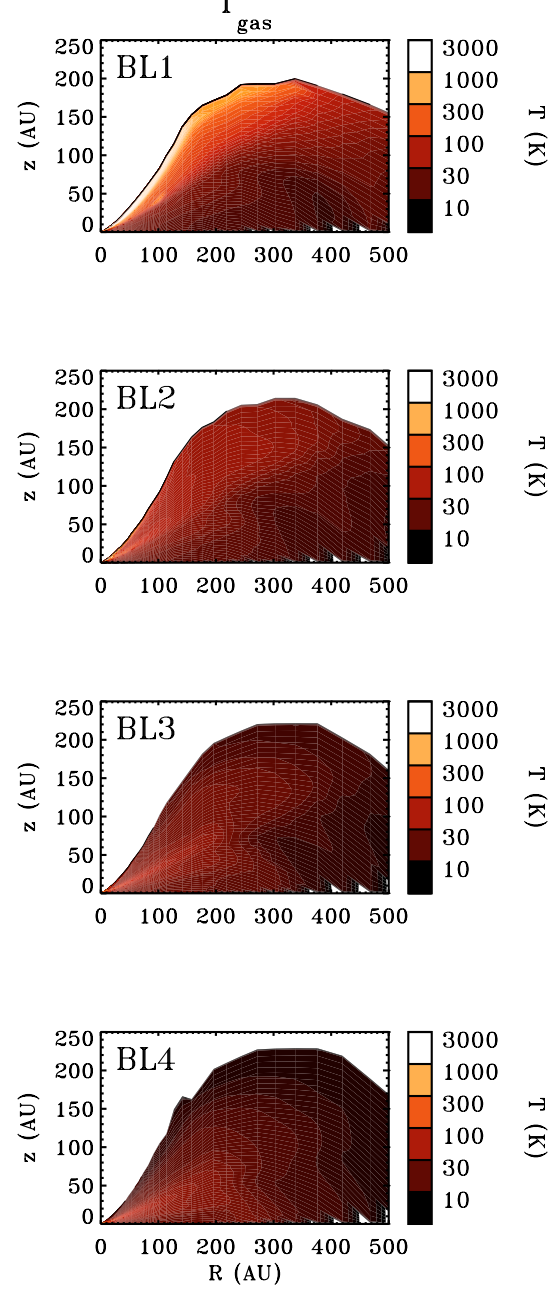

$\mathrm{CO}$
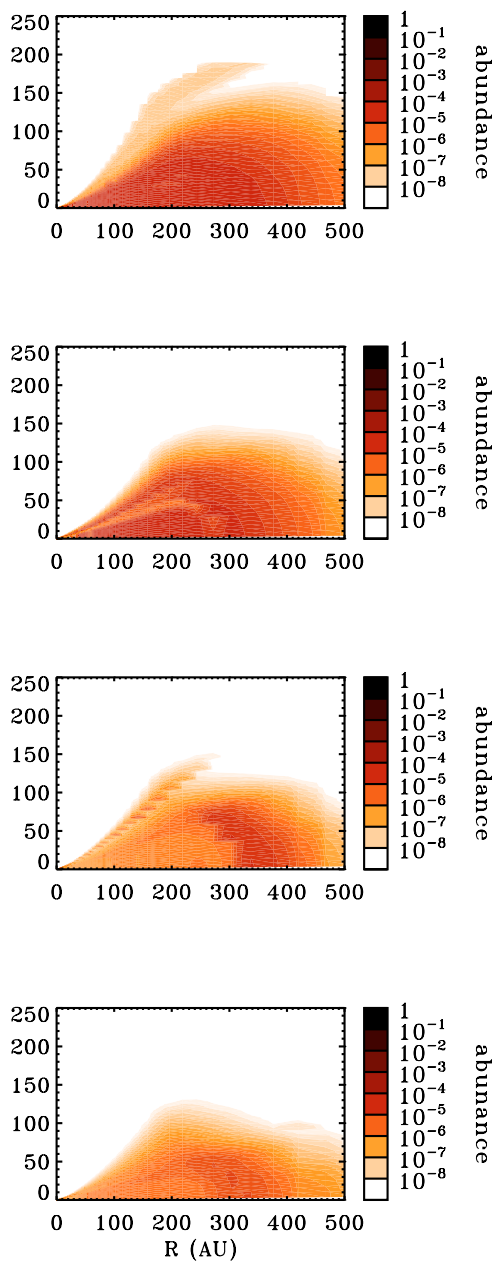

$\mathrm{HCN}$
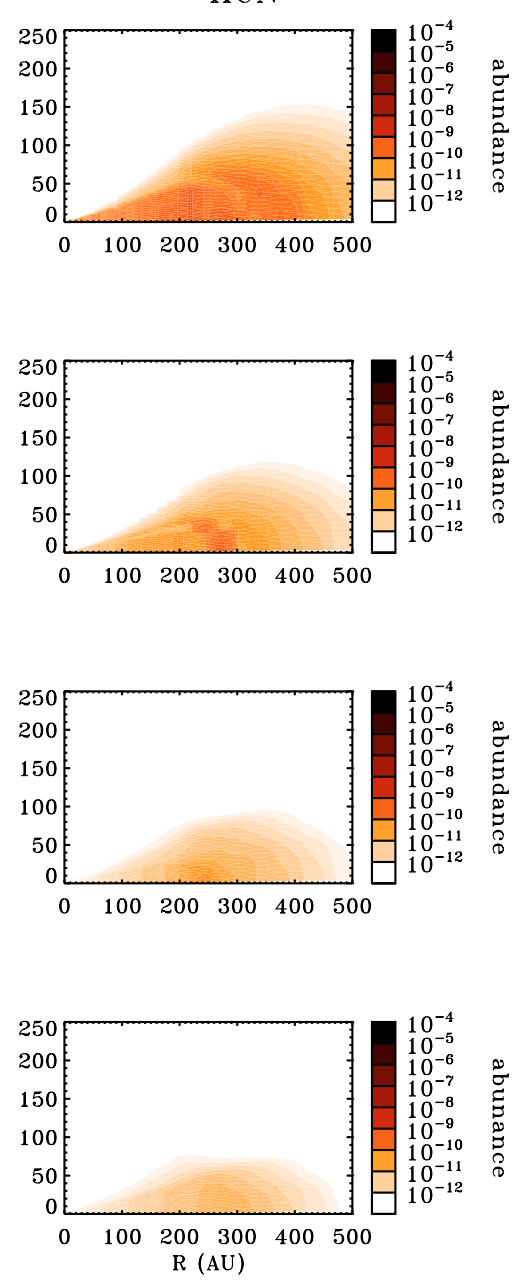

Fig. 5. Gas temperatures (left column), $\mathrm{CO}$ abundances (middle column) and HCN abundances (right column) for models BL1-BL4.

abundance in these models. In the BL series HCN occurs mainly in the midplane of the disk, in a layer that gets narrower going from BL1 to BL4. This explains the decreasing column densities in Fig. 8. The peaks in the vertical distributions of $\mathrm{CN}$ and $\mathrm{C}_{2} \mathrm{H}$ get broader going from BL1 to BL4, and consequently their column densities in the main portion of the disk $(R<200 \mathrm{AU})$ increase. In the outer disk the interstellar radiation field can dissociate these molecules, decreasing their column densities. The peak in the vertical $\mathrm{HCO}^{+}$distribution decreases in height going from model BL1 to BL4, which is reflected in the column densities.

The column densities of $\mathrm{CN}$ and $\mathrm{C}_{2} \mathrm{H}$ (and to a lesser extent those of $\mathrm{HCO}^{+}$) are relatively high in the outer parts of the disk $(R>200 \mathrm{AU})$. In spite of the fact that the total column density drops off as $R^{-12}$, the densities are still high enough to provide high abundances for these molecules. The abundances of these molecules in the outer disk depend strongly on the exact form of the total column density profile. If it drops off more rapidly than $R^{-12}$, this will be reflected in the intensities of the molecular lines. As a consequence, the emission lines of $\mathrm{CN}, \mathrm{C}_{2} \mathrm{H}$ and $\mathrm{HCO}^{+}$may be used to examine the outer regions of disks that are difficult to probe in the continuum.

The trends in the column densities of $\mathrm{CO}, \mathrm{CN}$ and $\mathrm{HCO}^{+}$ presented here for the BL series, in which dust growth and settling is simulated, show qualitative similarities to those found by Aikawa \& Nomura (2006) for T-Tauri disks with different stages of grain growth. With increasing grain size, they find that $N(\mathrm{CO})$ changes only very little, $N(\mathrm{CN})$ stays constant in the outer regions and increases in the inner regions, and $N\left(\mathrm{HCO}^{+}\right)$ decreases in the outer regions. They find no significant variation for $\mathrm{HCN}$ and $\mathrm{C}_{2} \mathrm{H}$, whereas column densities of these molecules change significantly in the current paper. This is possibly due to the jump in the stellar radiation field at $1200 \AA$, which has a strong influence on these molecules; Aikawa \& Nomura (2006) use a much smoother stellar spectrum that resembles the interstellar radiation field.

\subsection{Atomic and molecular lines}

From the molecular abundances and the gas temperature at each position in the disk the profiles of rotational molecular lines are calculated using the accelerated Monte Carlo code by Hogerheijde \& van der Tak (2000). The distance to the disk is taken to be $150 \mathrm{pc}$, and an inclination of $45^{\circ}$ is assumed. For the velocity field Keplerian rotation around a $2.5 M_{\odot}$ star and a turbulent velocity of $0.2 \mathrm{~km} \mathrm{~s}^{-1}$ were assumed. The data were convolved with a $6.7^{\prime \prime}$ beam, so that the disk fills the beam precisely. For molecules containing ${ }^{13} \mathrm{C}$ and ${ }^{18} \mathrm{O}$ the abundances of the main isotopic variant were used, scaled with factors of $1 / 45$ and $1 / 500$, respectively. This assumption does not hold everywhere in the disk due to isotope selective photodissociation, but the effects on integrated intensities over the entire disk 

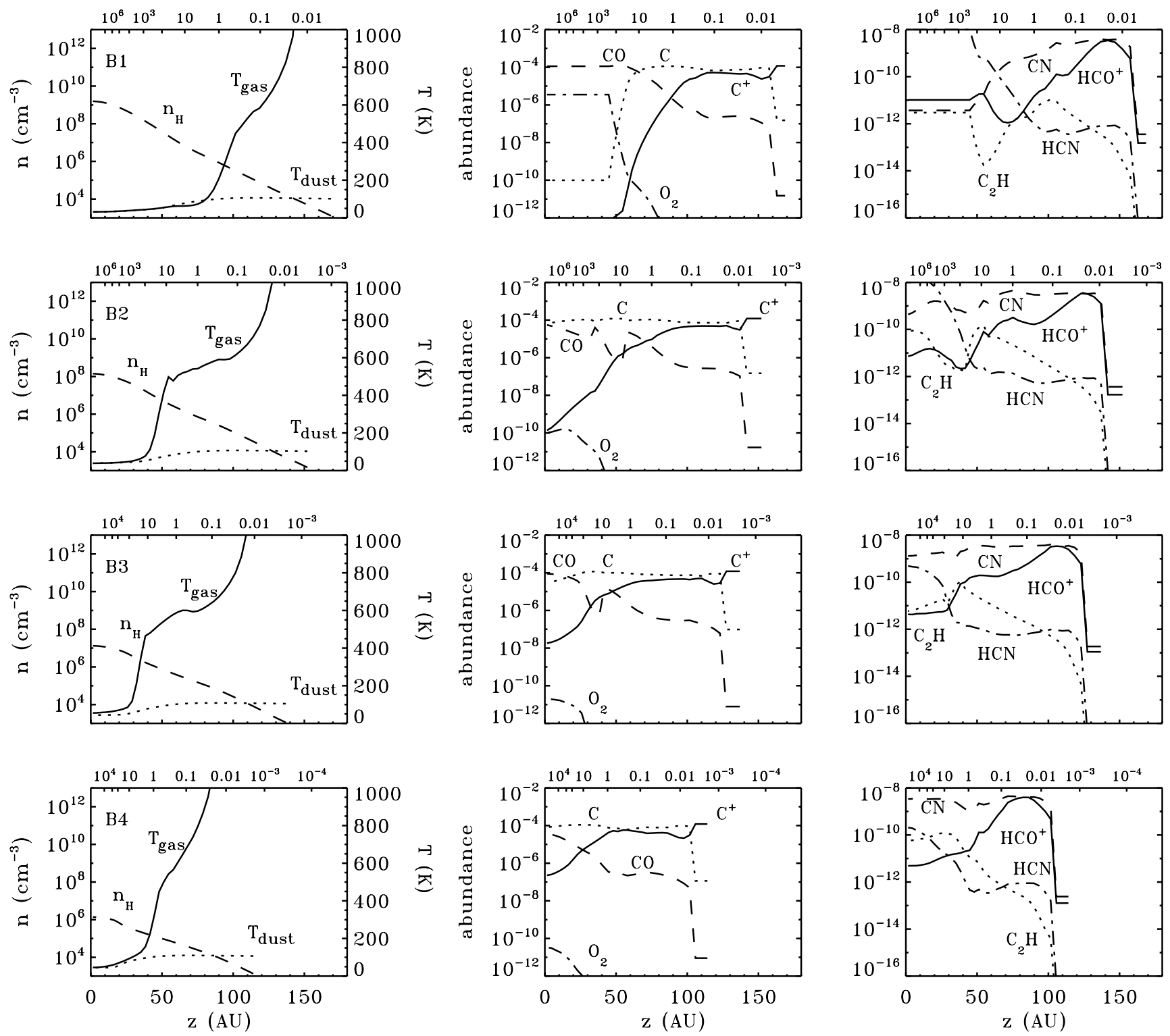

Fig. 6. Vertical profiles at a radius of $200 \mathrm{AU}$ of the gas temperature and density (left column) and chemistry (middle and right columns) for models B1-B4. The visual extinction toward the central star is given at the top of each panel.

are likely small. The resulting emission line profiles for $\mathrm{O}, \mathrm{CO}$, $\mathrm{HCN}$ and $\mathrm{CN}$ for models B1/BL1, B4 and BL4 are shown in Fig. 9.

It can be seen that the $[\mathrm{O}$ I] line does not change much when the mass of the disk decreases; the difference in intensity between models B1 and B4 is only a factor of a few, while the mass changes with a factor of 1000 . Model BL4 has a slightly higher $[\mathrm{O} \mathrm{I}]$ intensity, since at the temperatures where $\mathrm{O}$ can be excited, less oxygen is in the form of $\mathrm{CO}$ and $\mathrm{O}_{2}$. Also, the [O I] line has broader wings since it is excited mostly in the inner regions of the disk compared to model B1.

The CO $J=6-5$ line follows similar trends: it is optically thick in all models, making it relatively insensitive to total disk mass. The line is significantly broader in model B4 than in models B1 and BL4 because the CO column densities are more centrally concentrated in that model.

The HCN $J=4-3$ line shows a strong dependence on the disk mass. Model B4 has very low HCN abundances, giving this line an intensity in the $\mathrm{mK}$ range. The differences between models B1 and BL4 are less pronounced and reflect the slightly lower $\mathrm{HCN}$ abundances in BL4 with respect to B1. The CN $J=3-2$ line, however, shows a strong dependence of the line shape on the dust/gas ratio. This line has a relatively low excitation temperature $(\sim 30 \mathrm{~K})$, and the gas temperature in the region where it can be excited (i.e. where the density is equal to the critical density of $10^{7} \mathrm{~cm}^{-3}$ ) is too high in model BL4 to get an appreciable population in the upper level. In model B1 the temperatures are lower due to the decreased $\mathrm{C}$-ionization heating rate, and the $J=3$ level has a higher population. Model B4 only has a weak line here due to the low gas content of the disk.

The integrated intensities are presented in Fig. 10. It can be seen that model B1/BL1 generally produces strong emission lines, especially for the fine-structure transitions of $\mathrm{O}$ and the rotational lines of $\mathrm{CO}$. These lines are the main coolants of the PDR surface of the disk; therefore they radiate all the energy that is delivered to the gas via the various heating processes.

The $[\mathrm{CI}]$ intensities show an initial increase in the $\mathrm{B}$ series even though the disk mass decreases by a factor 10 , this is due 

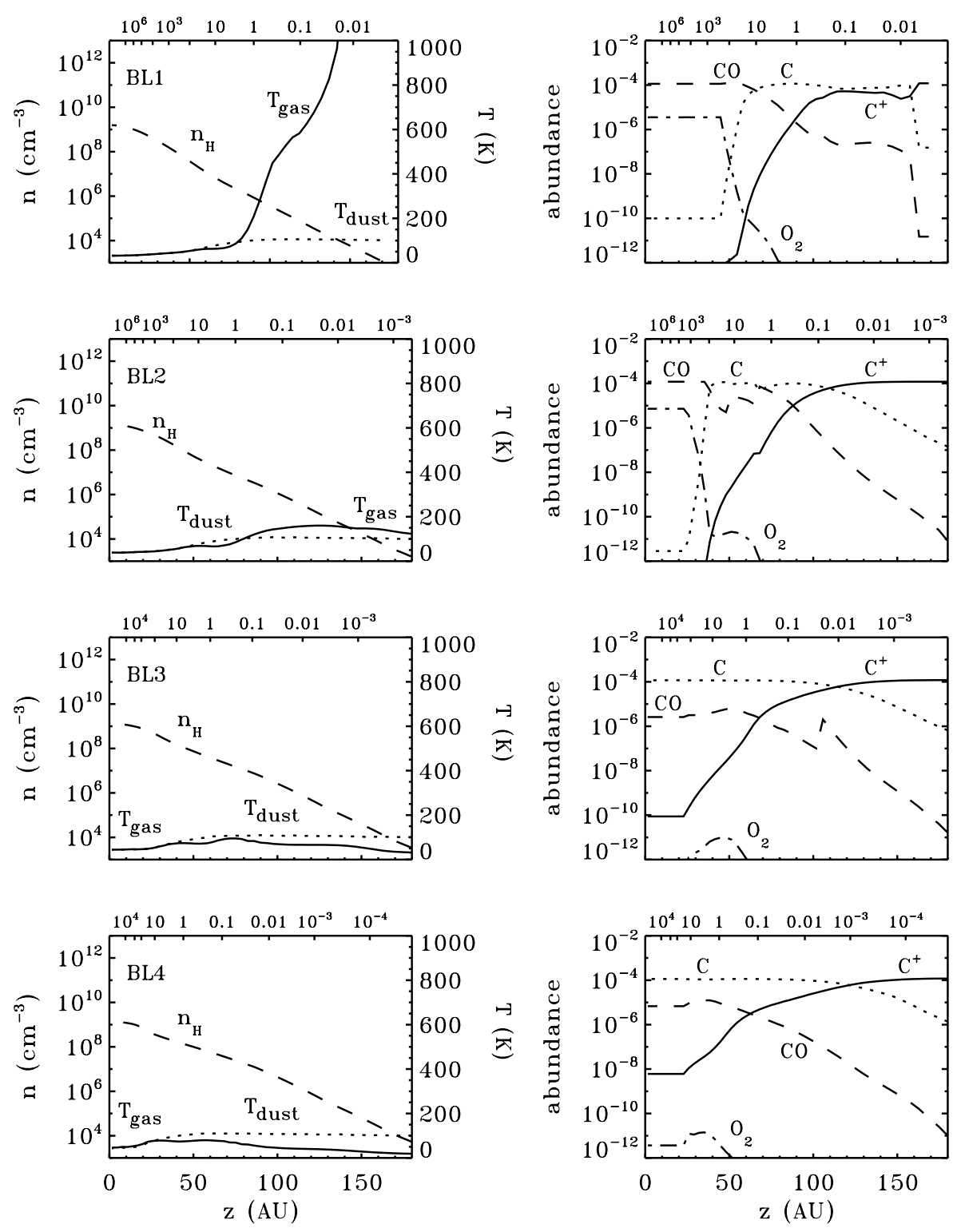
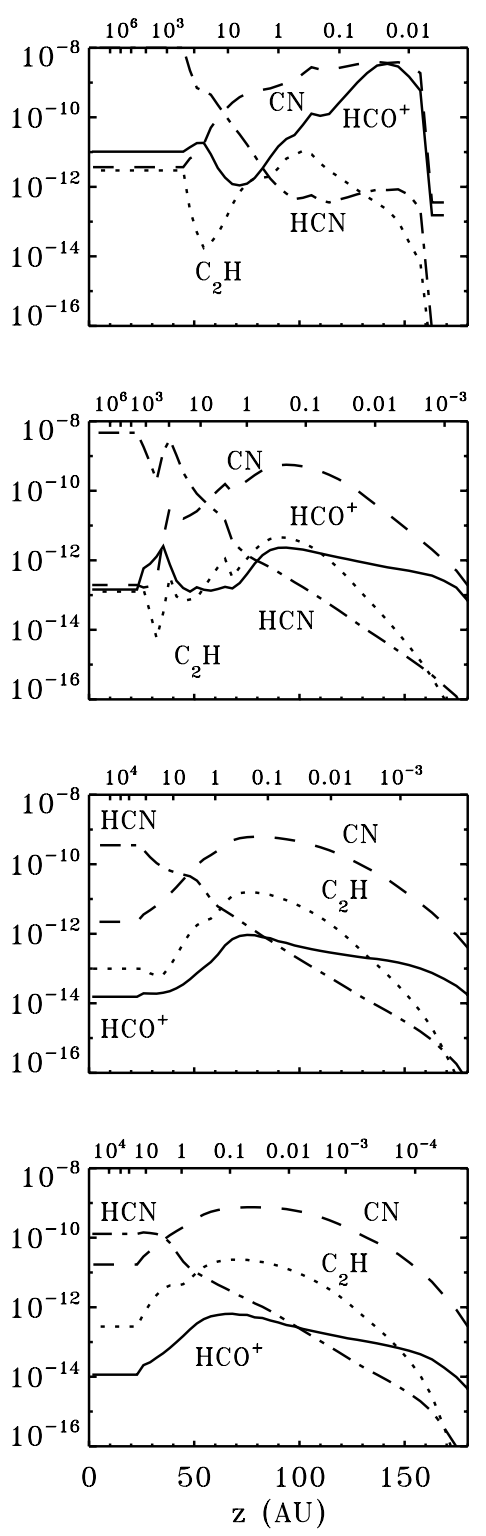

Fig. 7. As Fig. 6, but for models BL1-BL4.

to the high $\mathrm{C}$ abundance in model B2 compared to B1. In models B3 and B4 the intensity drops again, albeit slowly due to the optical thickness of the lines. In the BL series the [CI] intensities only increase due to the ever increasing $\mathrm{C}$ abundances in this series. Similar effects also determine the intensities of the [O I] line.

The [C II] fine-structure line is significantly weaker than those of $[\mathrm{OI}]$ and $[\mathrm{CI}]$, even though it is an important cooling line in PDRs. This line has a low critical density $\left(10^{3} \mathrm{~cm}^{-3}\right)$, which means is can only be an effective coolant at the immediate surface of the disk. Deeper into the disk this line is quenched by collisions. Its intensity increases in the BL series because the diffuse upper layers are thicker there.

The intensities of the $\mathrm{CO}$ rotational lines show a weak dependence on disk mass. Initially these lines are optically thick, but become optically thin going from model B3 to B4, causing a sudden drop in intensity. The lines do not change significantly in the BL series.

The pure rotational lines of $\mathrm{HCO}^{+}, \mathrm{HCN}$ and $\mathrm{CN}$ follow the same trends as CO: they change only little in the first steps of the B series, but become optically thin going from model B2 to $\mathrm{B} 3$ due to their lower abundance compared to $\mathrm{CO}$. These lines change very little in the $\mathrm{BL}$ series, with the exception of $\mathrm{HCO}^{+}$, which has a steadily decreasing column density in this series.

\subsection{Comparison with observations}

The rotational lines of $\mathrm{CO}, \mathrm{HCO}^{+}, \mathrm{CN}$ and $\mathrm{HCN}$ of two Herbig Ae stars, HD 163296 and MWC 480, have been observed by Thi et al. (2004) with IRAM and JCMT. The SEDs of these objects indicate that they are group II sources, meaning that of the models presented here only steps 3 or 4 of each series need to be considered. Correcting for beam dilution, their intensities of $\mathrm{CO},{ }^{13} \mathrm{CO}$ and $\mathrm{CN}$, as well as the $\mathrm{CO} /{ }^{13} \mathrm{CO}$ line ratio, fit well to the values found for model B2 (i.e. a $0.01 M_{\odot}$ disk with a dust/gas ratio of 0.01) with the HD 163296 data indicating a slightly higher gas mass than MWC 480. A good fit is also given by models BL3 and BL4 (i.e. a $0.1 M_{\odot}$ disk with a dust/gas ratio of $10^{-4}-10^{-5}$ ), with the HD 163296 data indicating a slightly lower dust/gas ratio than MWC 480. The masses agree 

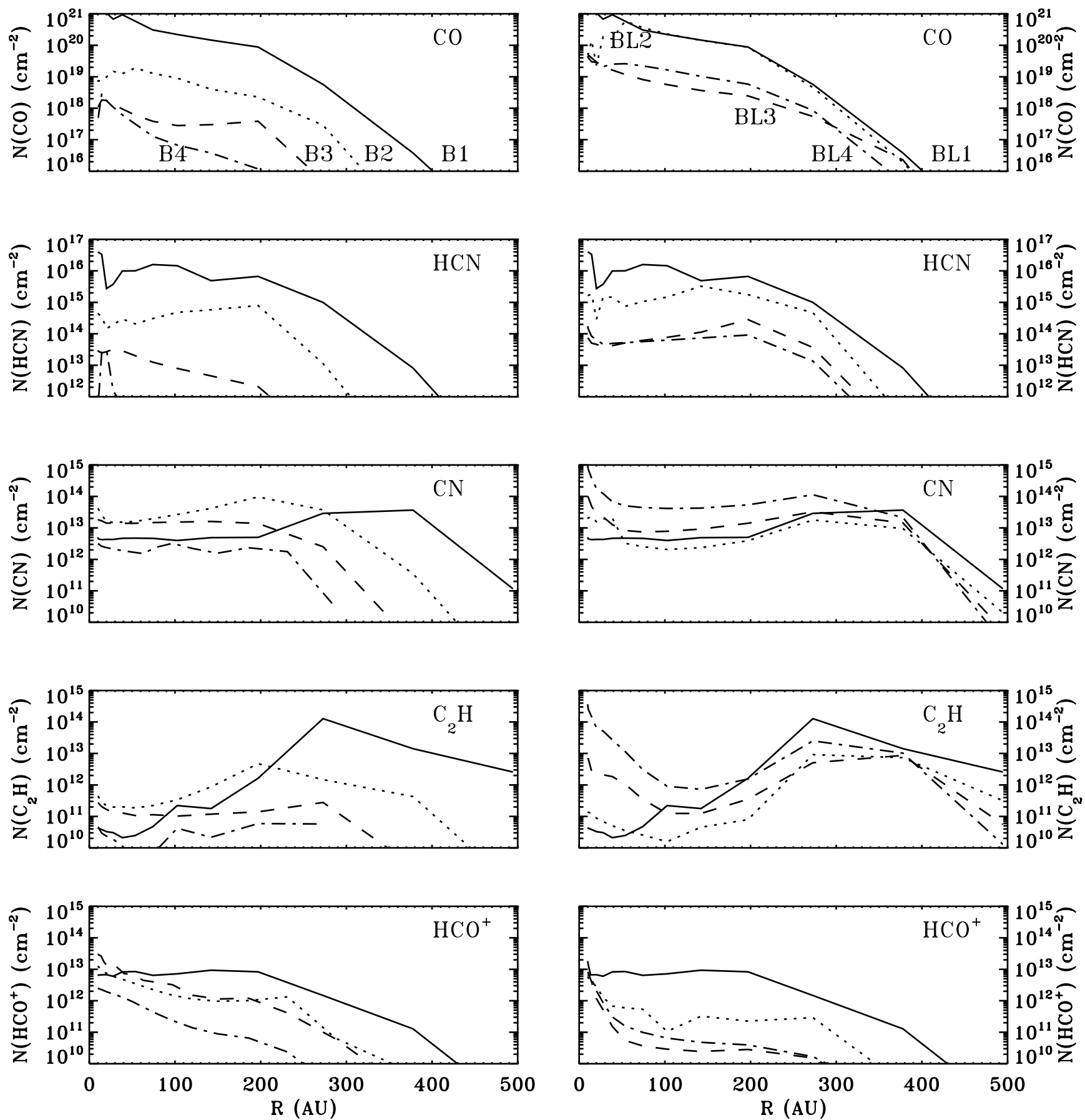

Fig. 8. The vertical column densities of $\mathrm{CO}$ (top row), $\mathrm{HCN}$ (second row), $\mathrm{CN}$ (third row), $\mathrm{C}_{2} \mathrm{H}$ (fourth row) and $\mathrm{HCO}^{+}($bottom row) for the $\mathrm{B}$ series (with a descending disk mass, left column) and the BL series (with a descending dust/gas ratio, right column). The solid lines give the results for model B1/BL1; the dotted line for B2/BL2; the dashed line for B3/BL3; the dash-dotted line for B4/BL4.

within a factor of a few with those derived from millimeter continuum observations assuming a dust/gas ratio of 0.01 . None of the models presented here can reproduce the $\mathrm{HCO}^{+}$lines by Thi et al. (2004), which have intensities of $>1 \mathrm{~K} \mathrm{~km} \mathrm{~s}^{-1}$. This may be due to the low $\mathrm{HCO}^{+}$abundances found here compared to other disk chemistry models. Since these objects are group II sources, it is unlikely that model B2 describes their structure. Instead, models BL3 and BL4 provide a good fit to the observational data.

The intensities predicted here for the $\mathrm{HCN}$ 4-3 line of models B2, BL3 and BL4 $\left(\sim 5 \mathrm{~K} \mathrm{~km} \mathrm{~s}^{-1}\right)$ exceed the upper limits by Thi et al. (2004) of 0.8 and $0.3 \mathrm{~K} \mathrm{~km} \mathrm{~s}^{-1}$ for HD 163296 and
MWC 480, respectively (corrected for beam dilution). This discrepancy may be explained by the fact that our models take freeze-out of molecules into account only by adjusting the global gas-phase elemental abundances, meaning that there is relatively little oxygen (since most of the dust is colder than the water sublimation temperature of $\sim 100 \mathrm{~K}$ ) compared to carbon (since all dust is warmer than the $\mathrm{CO}$ sublimation temperature of $\sim 20 \mathrm{~K}$ ). For nitrogen-bearing species this method is less accurate, however, since molecules that occur mostly in the disk's surface layers (like $\mathrm{CN}$ ) will not freeze out due to the high dust temperatures there, while molecules that occur mostly in the deeper regions near the midplane (like $\mathrm{HCN}$ ) are more likely to freeze 

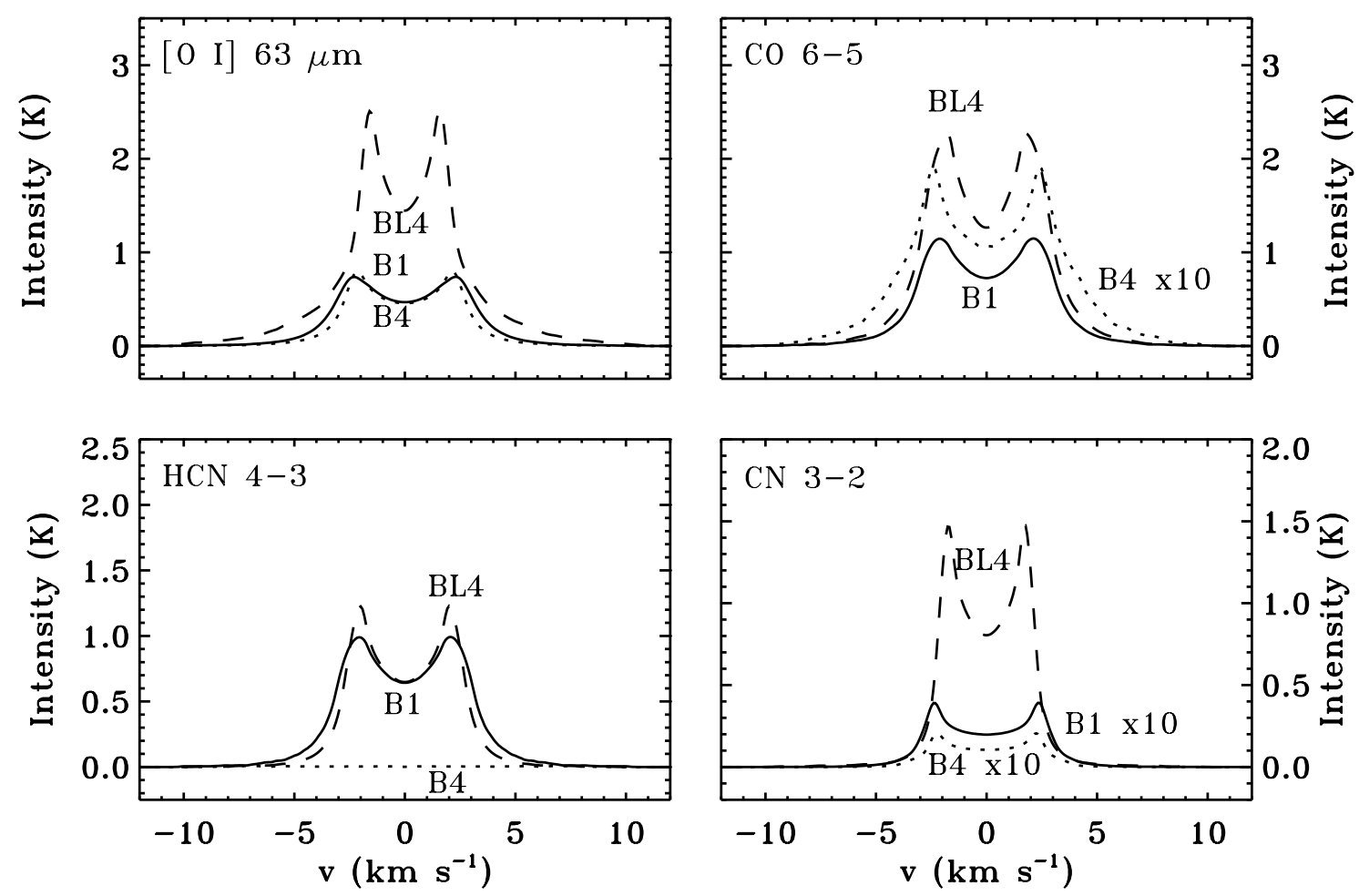

Fig. 9. Emission line profiles for O (top left), CO (top right), HCN (lower left) and CN (lower right) for models B1/BL1 (solid line), B4 (dotted line) and BL4 (dashed line).

out on the cold dust grains. To estimate the effect of freeze-out, the intensities were re-calculated by removing the HCN from regions where $T_{\text {dust }}<70 \mathrm{~K}$ (consistent with the TPD experiments on $\mathrm{H}_{2} \mathrm{~S}$ and $\mathrm{C}_{2} \mathrm{H}_{2}$ by Collings et al. 2004). The results are given in Fig. 11. It can be seen that freeze-out may account for more than an order of magnitude difference in intensity, and that the revised B2 model intensity is just consistent with the observed upper limits. The $\mathrm{CN}$ lines are less affected by freeze-out, since the peak in the vertical $\mathrm{CN}$ distribution occurs at larger heights than the HCN distribution, where the higher dust temperatures and lower densities will prevent freeze-out.

The intensities presented here indicate that future instruments like ALMA will be able to detect and image the rotational lines of $\mathrm{CO},{ }^{13} \mathrm{CO}$ and $\mathrm{CN}$ in disks with masses as low as $10^{-4} M_{\odot}$, with a typical sensitivity of $0.3 \mathrm{~K} \mathrm{~km} \mathrm{~s}^{-1}$ in $1 \mathrm{~h}$. The lines of $\mathrm{C}^{18} \mathrm{O}, \mathrm{HCO}^{+}$and $\mathrm{HCN}$ can also be imaged in these lowmass disks, but will require long integration times. Massive disks that undergo dust growth and settling will be readily observable, since the molecular column densities remain high even when the disk becomes more and more optically thin in the continuum.

There are several line ratios that can be used to ascertain whether a group II source (as determined by its SED) is the product of mass loss (putting it in the B series) or of dust growth and settling (putting it in the BL series). Some relevant ratios are plotted in Fig. 12: a low ratio $(<10)$ of $\mathrm{CO} /{ }^{13} \mathrm{CO}(<10)$ or $\mathrm{CO} / \mathrm{C}^{18} \mathrm{O}$ would indicate an object with a low dust/gas ratio instead of a low overall mass; ${ }^{12} \mathrm{C}^{16} \mathrm{O}$ is optically thick (thus making its lines relatively insensitive to the disk mass), while the isotopic variants of $\mathrm{CO}$ are optically thin, so they trace the $\mathrm{CO}$ abundance directly. ${ }^{13} \mathrm{CO} 3-2 / \mathrm{HCO}^{+} 4-3$ is another suitable tracer, since the $\mathrm{HCO}^{+}$intensities behave similarly in the $\mathrm{B}$ and $\mathrm{BL}$ series; on the other hand, the intensities of ${ }^{13} \mathrm{CO}$ drop off if the disk mass is decreased, while they remain high in the case of dust growth/settling. A high ratio $(>100)$ would indicate dust growth and settling, while a low ratio would indicate mass loss. The ratio of [O I] $63 \mu \mathrm{m} / 146 \mu \mathrm{m}$ directly probes the temperature structure of the upper layes, and is therefore suitable to distinguish between the B and BL series. A high ratio $(>2)$ would indicate that a disk with a dust/gas ratio close to 0.01 ; a lower ratio would indicate dust growth or settling. The ratio of [C I] $610 \mu \mathrm{m} /{ }^{13} \mathrm{CO} 3-2$ can be used to distinguish group I sources (B1-B2 and BL1-BL2) from group II sources (B3-B4 and BL3BL4), where a high ratio $(>5)$ would indicate a group II source. This ratio is not very sensitive to the differences between the $\mathrm{B}$ and BL series, however. The $\mathrm{CO} 6-5 / 2-1$ ratio was found to be insensitive to the differences between the B and BL series, since the gas temperatures stay high enough to excite the $J=6$ even in models with a very low dust/gas ratio (e.g. model BL4).

\section{Conclusions}

The thermal balance, chemistry and emission line profiles were calculated for a series of disk structure models representing different classes of protoplanetary disks around Herbig Ae stars with systematically decreasing disk masses or dust/gas ratios. The following can be concluded:

- For an interstellar dust/gas ratio (0.01), the gas temperatures at the flaring edge of the disk are very high, reaching temperatures of several thousand K. Directly underneath this hot surface layer is a warm (several hundreds of K) optically thin layer where the gas is heated directly by the stellar radiation. This warm layer drives an endothermic chemistry resulting in a relatively high $\mathrm{CO}$ abundance. In the midplane and in the outer disk the self-shadowing of the disk results in lower gas temperatures, usually close to the local dust temperature. This relatively cool self-shadowed part decreases in size with increasing disk mass. 


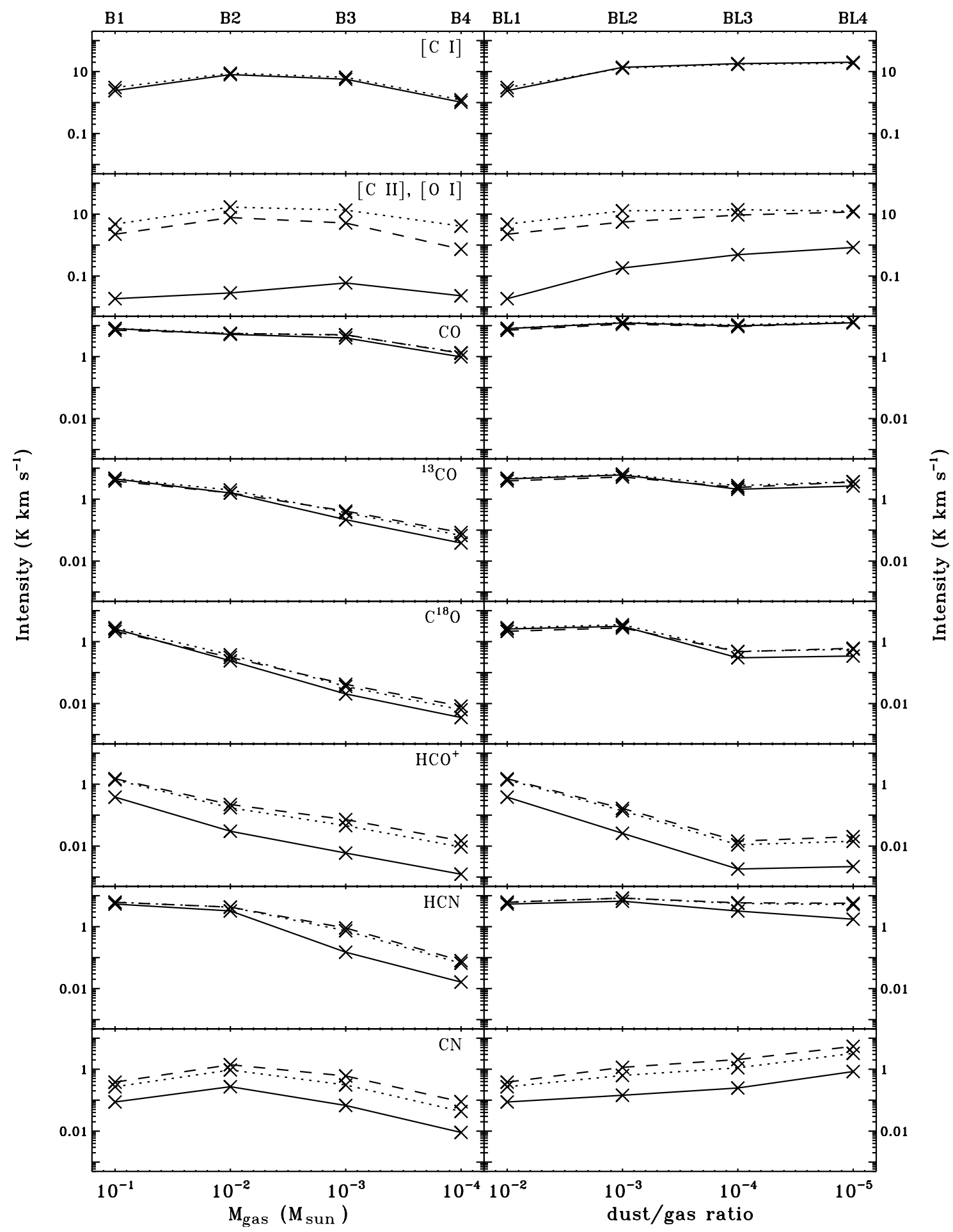

Fig. 10. Integrated intensities in a 6.7" beam for the B series (left column) and the BL series (right column). The top row gives the [CI] fine structure lines $(J=1-0$ solid, $J=2-1$ dotted). The second row gives the fine structure lines of [C II] fine structure line (solid) and [O I] $(J=1-2$ dotted, $J=0-1$ dashed). The third, fourth and fifth rows give the rotational lines of $\mathrm{CO},{ }^{13} \mathrm{CO}$ and $\mathrm{C}^{18} \mathrm{O}(J=2-1$ solid, $J=3-2$ dotted, $J=6-5$ dashed). The sixth row gives the rotational lines of $\operatorname{HCO}^{+}(J=1-0$ solid, $J=3-2$ dotted, $J=4-3$ dashed). The seventh row gives the rotational lines of HCN ( $J=1-0$ solid, $J=3-2$ dotted, $J=4-3$ dashed). The bottom row gives the rotational lines of CN $(J=1-0$ solid, $J=2-1$ dotted, $J=3-2$ dashed).

- The disk chemistry shows a strong dependence on disk mass. The low-mass disks have large optically thin atmospheres where fragile species like $\mathrm{C}_{2} \mathrm{H}$ and $\mathrm{HCN}$ are easily destroyed by $\mathrm{UV}$ radiation. More robust species like $\mathrm{CO}$ and $\mathrm{CN}$ still have relatively high abundances here.
- Although the self-shielding of CO is stronger in the BL series compared to the $\mathrm{B}$ series, the abundance of this molecule is lower. This is due to the reduced efficiency in $\mathrm{CO}$ formation in the absence of PAHsand classical grains, and the high photodissociation rates of $\mathrm{CO}$ precursors like $\mathrm{CH}$. 


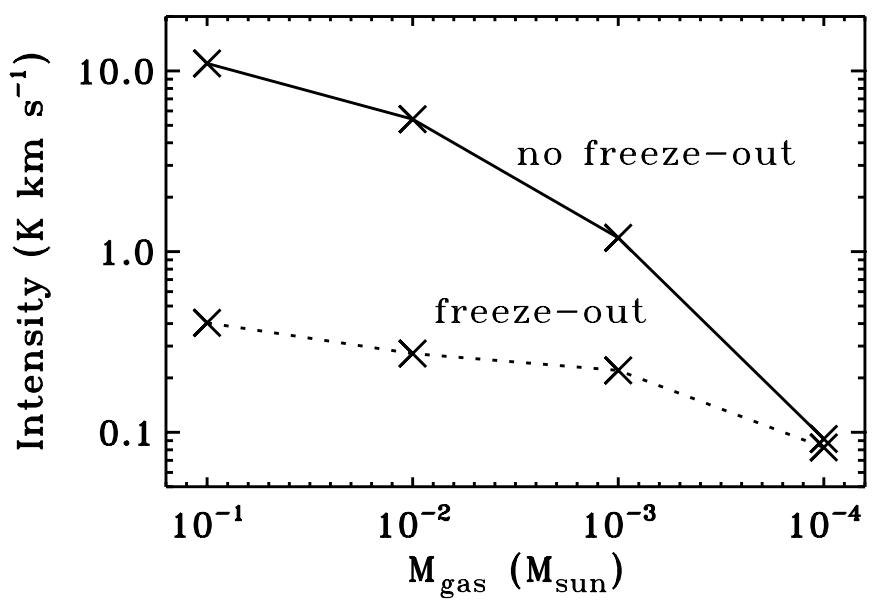

Fig. 11. Integrated intensities of $\mathrm{HCN}$ in the $\mathrm{B}$ series when all $\mathrm{HCN}$ is in the gas phase (solid line) and when $\mathrm{HCN}$ is removed from the gas phase for $T_{\text {dust }}<70 \mathrm{~K}$ (dotted line). The BL series follows similar trends.
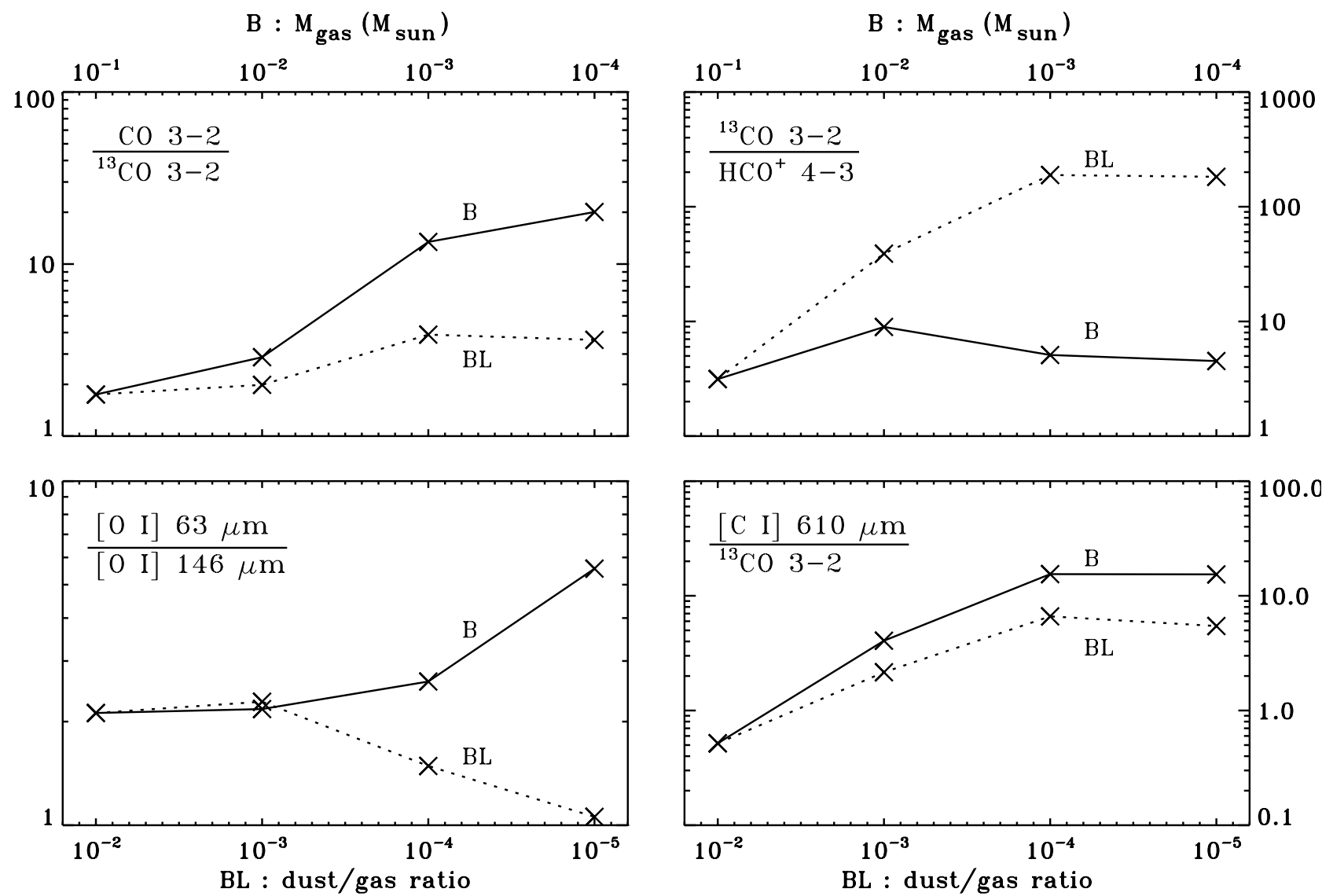

Fig. 12. Line ratios for the B series (solid lines) and the BL series (dotted lines).

- Dust settling has little effect on molecular abundances if the total gas mass is kept constant: there is only a small shift of the maximum abundance towards the midplane. The primary effect of dust settling on the disk is to lower the gas temperature by decreasing the importance of the photoelectric heating process.

- The CO rotational lines can be used to trace the total disk mass only for low-mass disks. In high-mass disks these lines are optically thick and not sensitive to the disk mass. The isotopic variants of $\mathrm{CO}\left({ }^{13} \mathrm{CO}\right.$ and $\left.\mathrm{C}^{18} \mathrm{O}\right)$ are much more sensitive even at high disk masses; their intensities are also mildly sensitive to the dust/gas ratio.

- Good tracers to distinguish between the B series and the BL series (i.e. whether a class II source is undergoing mass loss or dust growth and settling) are $\mathrm{CO} /{ }^{13} \mathrm{CO}$ and $\mathrm{CO} / \mathrm{C}^{18} \mathrm{O}$ line ratios, since the isotopic variants of $\mathrm{CO}$ are more sensitive to the total amount of $\mathrm{CO}$ in the disk than the main form. Also, the ${ }^{13} \mathrm{CO} 3-2 / \mathrm{HCO}^{+} 4-3$ and the [O I] $63 \mu \mathrm{m} / 146 \mu \mathrm{m}$ ratios can provide information on whether a source belongs in the $\mathrm{B}$ or the $\mathrm{BL}$ series. $[\mathrm{CI}] /{ }^{13} \mathrm{CO}$ can be used to distinguish group I from group II sources, but is not sensitive to 
the differences between a disk undergoing mass loss or dust growth and settling.

- In the disk models presented here, the dust temperatures are high enough to prevent the freeze-out of $\mathrm{CO}$ onto dust grains. For other molecules, like HCN, freeze-out remains possible, but has not been included explicitly in the current code.

Acknowledgements. The authors are grateful to Inga Kamp and Xander Tielens for their helpful comments. This work was supported by a Spinoza grant from the Netherlands Organisation for Scientific Research and by the European Community's Human Potential Programme under contract HPRN-CT-200200308, PLANETS.

\section{References}

Acke, B., \& van den Ancker, M. E. 2004, A\&A, 426, 151

Aikawa, Y., \& Nomura, H. 2006, ApJ, accepted

Aikawa, Y., Umebayashi, T., \& Miyama, S. M. 1997, ApJ, 486, L51

Bakes, E. L. O., \& Tielens, A. G. G. M. 1994, ApJ, 427, 822

Bary, J. S., Weintraub, D. A., \& Kastner, J. H. 2003, ApJ, 586, 1136

Brittain, S. D., Rettig, T. W., Simon, T., et al. 2003, ApJ, 588, 535

Canosa, A., Laubé, S., Pasquerault, D., Gomet, J. C., \& Rowe, B. R. 1995, Chem. Phys. Lett., 245, 407

Chiang, E. I., \& Goldreich, P. 1997, ApJ, 490, 368

Collings, M. P., Anderson, M. A., Chen, R., et al. 2004, MNRAS, 354, 1133

D’Alessio, P., Cantó, J., Calvet, N., \& Lizano, S. 1998, ApJ, 500, 411

Dent, W. R. F., Greaves, J. S., \& Coulson, I. M. 2005, MNRAS, 359, 663

Draine, B. T. 1978, ApJS, 36, 595

Dullemond, C. P., \& Dominik, C. 2004, A\&A, 417, 159
Dullemond, C. P., van Zadelhoff, G.-J., \& Natta, A. 2002, A\&A, 389, 464

Dutrey, A., Guilloteau, S., \& Guélin, M. 1997, A\&A, 317, L55

Gorti, U., \& Hollenbach, D. 2004, ApJ, 613, 424

Habart, E., Natta, A., \& Krügel, E. 2004, A\&A, 427, 179

Hauschildt, P. H., Allard, F., \& Baron, E. 1999, ApJ, 512, 377

Hogerheijde, M. R., \& van der Tak, F. F. S. 2000, A\&A, 362, 697

Jansen, D. J., van Dishoeck, E. F., Black, J. H., Spaans, M., \& Sosin, C. 1995, A\&A, 302, 223

Jonkheid, B., Faas, F. G. A., van Zadelhoff, G.-J., \& van Dishoeck, E. F. 2004, A\&A, 428, 511

Jonkheid, B., Kamp, I., Augereau, J.-C., \& van Dishoeck, E. F. 2006, A\&A, 453, 163

Kamp, I., \& Dullemond, C. P. 2004, ApJ, 615, 991

Kamp, I., Dullemond, C. P., Hogerheijde, M., \& Enriquez, J. E. 2006, in Proc. IAU Symp. 231, Astrochemistry: Recent Successes and Current Challenges, ed. D. C., Lis, G. A. Blake, \& E. Herbst (Cambridge: Cambridge University Press), 377

Kamp, I., \& van Zadelhoff, G.-J. 2001, A\&A, 373, 641

Kamp, I., van Zadelhoff, G.-J., van Dishoeck, E. F., \& Stark, R. 2003, A\&A, 397, 1129

Kenyon, S. J., \& Hartmann, L. 1987, ApJ, 323, 714

Koerner, D. W., \& Sargent, A. I. 1995, AJ, 109, 2138

Nomura, H., \& Millar, T. J. 2005, A\&A, 438, 923

Spaans, M., Tielens, A. G. G. M., van Dishoeck, E. F., \& Bakes, E. L. O. 1994, ApJ, 437, 270

Thi, W. F., van Dishoeck, E. F., Blake, G. A., et al. 2001, ApJ, 561, 1074

Thi, W.-F., van Zadelhoff, G.-J., \& van Dishoeck, E. F. 2004, A\&A, 425, 955

Tielens, A. G. G. M., \& Hollenbach, D. J. 1985, ApJ, 291, 722

van Dishoeck, E. F. 1988, in Rate Coefficients in Astrochemistry, ed. T. J. Millar, \& D. A. Williams (Dordrecht: Kluwer Academic Publishers), ASSL 14649 van Dishoeck, E. F., \& Black, J. H. 1982, ApJ, 258, 533

van Zadelhoff, G.-J., Aikawa, Y., Hogerheijde, M., \& van Dishoeck, E. F. 2003, A\&A, 397, 789 